\section{Christoph Bachmann}

\section{Einführung}

Die Aromatherapie ist eine spezielle Art der Phytotherapie, die wiederum die älteste Therapierichtung der Menschheit darstellt. Schon in Gräbern von Neandertalern wurden Kräuterbeigaben gefunden, die allenfalls zu Therapiezwecken oder aus kultischen Gründen beigelegt wurden.

Bei allen alten Kulturen - wie in Mesopotamien, bei den Induskulturen, im alten Ägypten und später in der arabischen Welt - waren Kräuter und Düfte zu Heilzwecken oder bei kultischen Handlungen bekannt.

Hippokrates, Hildegard von Bingen, Paracelsus und viele weitere Heilkundige führten diese Tradition weiter und bauten sie aus. Samuel Hahnemann hat ebenfalls immer mit ätherischen Ölen gearbeitet und darf auch als Aromatherapeut bezeichnet werden. Ihm verdanken wir den Satz: «Das Riechen ist der Königsweg zur Seele.»

Als eigentlicher Begründer der Aromatherapie gilt aber der französische Chemiker und Parfümeur René-Maurice Gattefossé, der in der ersten Hälfte des 20. Jahrhunderts umfangreiche Studien unternahm und diverse Produkte mit ätherischen Ölen entwickelte.

\section{Anwendung}

Sehr interessant ist die andersartige Anwendung der Aromatherapie in verschiedenen Ländern: In Frankreich werden Aromatherapeutika vor allem eingenommen, dürfen jedoch nur von approbierten Ärzten verschrieben werden. Ganz anders sieht es in Gross-

\title{
Aromatherapie kann die Wirkung von Schüssler-Salzen optimieren
}

Der folgende Beitrag ist eine Zusammenfassung eines Referates, das Dr. med. Erwin P. Häringer, der in München eine Arztpraxis mit einem breiten Angebot an komplementärmedizinischen Behandlungsmethoden betreibt, im November in verschiedenen Schweizer Städten gehalten hat.

britannien und Deutschland aus: In Grossbritannien kennt man die Aromatherapie vor allem als Massagen und Bäder. In Deutschland hingegen werden die entsprechenden Präparate vor allem als Inhalationen angeboten und von vielen nichtärztlichen Therapeuten empfohlen.

\section{Riechen und limbisches System}

Bis etwa vor 20 Jahren galt der Geruchssinn als verborgener Sinn. Diese Auffassung hat sich seither aber grundlegend verändert. Die Geruchswahrnehmung des Menschen wurde intensiv erforscht. Etwa 380 Gene steuern die Bildung unserer etwa 350 Geruchsrezeptoren. Neue Forschungen belegen, dass der Mensch etwa 1 Billion Gerüche unterscheiden kann. Als Vergleich: Nur 3 Gene steuern unser Sehen, das uns erlaubt, maximal 150-200 verschiedene Farben unterscheiden zu können.

Weiter wurde entdeckt, dass das Riechen und das limbische System, dieser uralte Teil des Gehirns, eng zusammengehören. Gerüche können direkt in das limbische System gelangen und dort Wirkungen auslösen. Dies ist angesichts der Tatsache, dass viele Krankheiten einen psychischen Hintergrund haben, äusserst interessant.
Ausserdem ist die Geruchserinnerung des Menschen von grossem Interesse. Menschen in Extremsituationen, die äusserst erregt oder von panischer Angst ergriffen waren, wurden Düfte vorgeführt, die sie an ihre frühe Kindheit erinnerten. In sehr vielen Fällen beruhigten sich die Betroffenen sehr schnell.

\section{Ätherische öle}

Bei der Aromatherapie spielen ätherische Öle die entscheidende Rolle. Der Begriff Äther stammt vom griechischen Wort «Aither» ab, das als die Personifikation des Himmels galt. Nach Aristoteles ist er das fünfte Element. In der Alchemie war Äther als Sulfur dem Bewusstsein, dem feurigen Aspekt der Seele und den Lichtquanten zugeordnet.

Chemisch gesehen sind ätherische Öle charakteristische, stark duftende, volatile, lipophile sogenannte sekundäre Pflanzeninhaltsstoffe. Pflanzen synthetisieren ätherische Öle als:

- Energiespeicher,

- Krankheitsschutz vor Pilzen und Bakterien,

- Temperaturregelung,

- Anlockung oder Abschreckung von Tieren,

- Kommunikation von Pflanzen.

\section{KARGER}

(C) 2015 S. Karger GmbH, Freiburg

Fax +497614520714 
Tab. 1. Vorkommen von ätherischen Ölen in Pflanzenorganen

$\begin{array}{ll}\text { Blüte } & \text { z.B. Rose, Jasmin, Kamille } \\ \text { Blätter } & \text { z.B. Salbei, Melisse, Thymian } \\ \text { Wurzel } & \text { z.B. Kalmus, Angelika, Vetiver } \\ \text { Früchte } & \text { z.B. Anis, Kümmel, Koriander } \\ \text { Holz } & \text { z.B. Sandelholz } \\ \text { Rinde } & \text { z.B. Zimt } \\ \text { Harz } & \text { z.B. Weihrauch, Myrrhe } \\ \text { Schale } & \text { z.B. Agrumen }\end{array}$

Ätherische Öle sind also keine fetten Öle, trotzdem aber lipophil, extrem flüchtig und lichtempfindlich. Sie zeichnen sich bei guter Lagerung durch eine lange Haltbarkeit aus, ertragen aber keine Extremtemperaturen. Sie sind sehr gut mit fetten Ölen und Emulgatoren, aber nur zu einem geringen Mass mit Wasser mischbar.

Der Mensch kann nur Substanzen bis zu einem Molekulargewicht von etwa maximal 200 riechen. Darüber reicht der Dampfdruck nicht mehr aus, um einen entsprechenden Reiz auszulösen.

Ätherische Öle findet man in Öldrüsen in oder auf dem Pflanzengewebe in verschiedenen Organen (Tab. 1).

\section{Gewinnung von ätherischen Ölen}

Zur Gewinnung von ätherischen Ölen muss oft ein sehr grosser Aufwand betrieben und sehr viel Pflanzenmaterial verarbeitet werden. Für 1 $\mathrm{kg}$ des entsprechenden ätherischen Öls sind wie folgt notwendig:

- $160 \mathrm{~kg}$ Lavendel,

- 1000 kg Jasminblüten,

- 5000 kg Rosenblätter,

- die Schalen von 1000 Zitronen,

- 1000 kg Neroliblüten.

Die Gewinnung geschieht durch Enfleurage, Kaltpressung, Wasserdampfdestillation, Destraktion mit Kohlendioxid oder Extraktion mit Hexan.

Die Wasserdampfdestillation ist die gängigste Methode, bei der die ätherischen Öle mit Wasserdampf aus der Pflanze gelöst und in ein Auffanggefäss geleitet werden. Nach dem $\mathrm{Ab}$ kühlen werden das ätherische Öl und das Wasser getrennt. Diese Art der Gewinnung erfordert viel Wissen und Erfahrung, da Druck, Temperatur und Zeit die Qualität und Ausbeute enorm beeinflussen!

\section{Qualität und Reinheit}

Die Herkunft, das örtliche Mikroklima, der Anbau, der Erntezeitpunkt und die Technik der Gewinnung spielen für die Qualität der ätherischen Öle eine entscheidende Rolle. Verschiedene Arzneibuch-Monographien, z.B. die Monographie «Ätherische Öle» der Pharmacopoea Europaea, garantieren die Qualität und Reinheit der entsprechenden ätherischen Öle. Dabei sind die eben erwähnten Kriterien der botanischen Herkunft, des geographischen Ursprungs der Pflanze, der Anbau, die Zucht sowie die Gewinnung entscheidend für die Erfüllung der Anforderungskriterien.

\section{Beispiel eines ätherischen Öls: Bergamottöl}

Bergamottöl wird aus Citrus aurantium ssp (Subspecies), einer Kreuzung aus Bitterorange und Zitrone, gewonnen. Diese Pflanze wächst in Süditalien und wird geerntet, wenn die Früchte noch im unreifen Stadium sind. Die Öldrüsen befinden sich in der äusseren Schale. Aus $100 \mathrm{~kg}$ Schalen werden etwa $500 \mathrm{~g}$ Essenz durch Kaltpressen und Zentrifugieren gewonnen. Wichtige Inhaltsstoffe sind Linalylacetat, Terpene, Bergapten, Bergamottin (Furocumarin), Dihydrocuminalkohol, Nerol, D-Limonen, Bergaptol und Limettin. Der Geruch des Bergamottöls wird von den meisten Menschen als sehr angenehm empfunden

Bergamottöl ist ein Bestandteil des Kölnischwassers und wird weiter gegen depressive Verstimmungen, Angst und Stress eingesetzt. Die Wirkung erfolgt über das limbische System. Weiter kennt man auch seine antiseptische Wirkung als Gurgelwasser. Es erhöht ausserdem die Lichtempfindlichkeit der Haut.

\section{Wirkungsort und Wirkungsmechanismus}

Die Moleküle des ätherischen Öls gelangen durch die Nase zur Riechschleimhaut, wo sie an die Rezeptoren der Reizsinneshaare andocken. Dadurch wird der Geruchsreiz zum Riechkolben und dann zum limbischen System weitergeleitet. Das bedeutet, dass der Duft ohne «Zensur» durch das zentrale Nervensystem (ZNS) in das limbische System weitergeleitet wird. Man kann daher sagen, dass das ZNS durch die Riechschleimhaut einen direkten Kontakt zur Aussenwelt hat.

Im limbischen System verursachen die Duftreize eine Sekretion von Neurotransmittern, die wiederum Sympathie und Abneigung, Motivation, Stimmungen und Erinnerungen bewirken. Düfte werden als Erinnerung lebenslang gespeichert.

Weiter können sich ätherische Öle in niedrigen Konzentrationen, systemisch angewendet, in bestimmten Arealen der Zellmembran einlagern und dort lokalisierte Enzyme, Carrier, Ionenkanäle oder Rezeptoren beeinflussen.

In mittleren Konzentrationen haben sie einen membranstabilisierenden Effekt, ähnlich wie Lokalanästhetika.

In höheren Konzentrationen werden sie lokal angewendet und lösen durch Reizwirkung unspezifische Effekte aus.

\section{Anwendungsgebiete der ätherischen öle}

Ein wichtiges Anwendungsgebiet der ätherischen Öle ergibt sich aus sei- 


\begin{tabular}{ll}
\hline Schüssler-Salz/übliche Potenz & Anwendung für/zur \\
\hline Nr. 1 Calcium fluoratum D12 & Stütz- und Bindegewebe \\
Nr 2 Calcium phosphoricum D6 & Knochen und Zähne \\
Nr. 3 Ferrum phosphoricum D12 & $\begin{array}{l}\text { Immunsystem, durch Hyperämie bedingte Schmerzen } \\
\text { und Wunden }\end{array}$ \\
Nr. 4 Kalium chloratum D6 & Entzündungen der Schleimhäute \\
Nr. 5 Kalium phosphoricum D6 & Nerven und Psyche \\
Nr. 6 Kalium sulfuricum D6 & Entschlackung \\
Nr 7 Magnesium phosphoricum D6 & Muskeln und Nerven, «Heisse Sieben» \\
Nr 8 Natrium chloratum D6 & Regulation des Wasserhaushalts \\
Nr 9 Natrium phosphoricum D6 & Stoffwechsel (Säure-Basen-Haushalt) \\
Nr 10 Natrium sulfuricum D6 & innere Reinigung \\
Nr 11 Silicea D12 & Haut und Haare \\
Nr 12 Clacium sulfuricum D6 & Gelenke \\
\hline
\end{tabular}

ner antiviralen Wirksamkeit. Im Gegensatz zur Schulmedizin, die wenig überzeugende Strategien gegen Viren anzubieten hat, gibt es verschiedene ätherische Öle mit einem antiviralen Potenzial! Weiterhin kennt man antiseptische, fungizide, schleim- und krampflösende Wirkungen von ätherischen Ölen. Diese können auch zur Beruhigung und gegen depressive Verstimmung, zur Förderung der Durchblutung und zur Verbesserung des Immunsystems, zum Kühlen und zur Pflege der Haut eingesetzt werden.

\section{Schüssler-Salze}

Der Hahnemann-Schüler Dr. Wilhelm Heinrich Schüssler postulierte, dass die Ursache vieler Erkrankungen in einer Störung der Mineralstoffverteilung in den Zellen liegt. In der Folge entwickelte Schüssler 12 homöopathisch aufbereitete Salze zur Beeinflussung der Mineralstoffverteilung: die bekannten «Schüssler-Salze» oder biochemischen Salze (Tab. 2).

\section{Aromasprays und Schüssler-Salze}

Der Grundgedanke hinter der gleichzeitigen Anwendung von Schüssler-Salzen und ätherisch-ölhaltigen Aromasprays ist folgender: Die Wirkung der Schüssler-Salze kann durch Riechgaben unterstützt und intensiviert werden. Bereits Samuel Hahne- mann hat seine Patienten oft, besonders bei LM-Potenzen, an den homöopathischen Arzneimitteln riechen lassen und dies mit einem $R$ auf seinen Rezepten dokumentiert.

Ein aromatherapeutischer Arzt hat 12 Aromasprays für jedes SchüsslerSalz entwickelt, von denen jeder auf der Grundlage der ursprünglichen Indikationen von Wilhelm Heinrich Schüssler beruht. Ein Aromaspray verbessert die Aufnahme des entsprechenden Schüssler-Salzes und unterstützt seine Wirksamkeit.

Ein Beispiel: Schüssler-Salz und Piniol ${ }^{\circledR}$ Aromaspray Nr. 5

Der Piniol Aromaspray Nr. 5 besteht aus den ätherischen Ölen von $A n$ gelica, Neroli und Zeder, die aromatherapeutisch den Indikationsbereich von Kalium phosphoricum D6 widerspiegeln. Die Kombination kann gegen Erschöpfungszustände, Kräfteverfall, psychische Schwäche, hohes Fieber, depressive Verstimmung, Parodontose, Mundgeruch und muskuläre Funktionsstörungen eingesetzt werden.

Die in diesem Aromaspray enthaltenen ätherischen Öle zeigen bei folgenden Symptomen spezifische Wirkungen:

- Angelica: Gedächtnisschwäche, Angst, Weinerlichkeit, Muskelund Nervenschwäche, Lähmungsgefühl, depressive Verstimmung, allgemeine Schwäche.
- Neroli: Gedächtnisschwäche, Angst, Weinerlichkeit, Schlaflosigkeit, Krämpfe und allgemeine Schwäche.

- Zeder: Gedächtnisschwäche, Angst, Weinerlichkeit, Muskelund Nervenschwäche, Lähmungsgefühl, Schmerzen, Depression, Neurasthenie, hysterische Verstimmung, Schlaflosigkeit, Krämpfe, Schwäche.

\section{Anwendung der Piniol Aroma- sprays}

1 Sprühstoss enthält etwa $0,14 \mathrm{ml}$. Somit können mit einem Spray von 30 $\mathrm{ml}$ etwa 200 Sprühstösse abgegeben werden.

1-2 Sprühstösse werden in die Raumluft oder auf den in der Packung beiliegenden Duftanhänger abgeben. Die Anwendung kann nach Belieben des Patienten wiederholt werden. Der Duft ätherischer Öle hält je nach $\mathrm{Zu}$ sammensetzung bis zu mehrere Stunden an. Die Anwendungsdauer ist nicht begrenzt.

Die Aromasprays sollen nicht auf die Haut gesprüht werden, weil die Resorption durch die Haut nicht erwünscht ist und sie ausserdem Hautreizungen bewirken können. Ein Sprühen auf die Haut kann allenfalls auch die Lichtempfindlichkeit erhöhen.

Ein Aufbringen der Aromasprays auf andere Träger wie etwa Tücher 
Table 3. SchüsslerTrios und Aromasprays

\begin{tabular}{|c|c|c|c|}
\hline Trio & Indikation/Anwendung & Schüssler-Salz & Aromaspray \\
\hline Allergie & $\begin{array}{l}\text { Heuschnupfen, Allergien: Katzen, Lebensmittel, } \\
\text { Nahrungsmittel, Sonne, Waschmittel }\end{array}$ & $\begin{array}{l}\text { Nr. } 2 \text { Calcium phosphoricum } \\
\text { Nr. } 6 \text { Kalium sulfuricum } \\
\text { Nr. } 10 \text { Natrium sulfuricum }\end{array}$ & Nr. 2 \\
\hline Bindegewebe & $\begin{array}{l}\text { Bindegewebsschwäche, Cellulitis, Krampfadern, } \\
\text { faltige Haut, Hämorrhoiden, Haarausfall, } \\
\text { brüchige Nägel }\end{array}$ & $\begin{array}{l}\text { Nr. } 1 \text { Calcium fluoratum } \\
\text { Nr. } 2 \text { Calcium phosphoricum } \\
\text { Nr. } 11 \text { Silicea }\end{array}$ & Nr. 11 \\
\hline Energiebalance & $\begin{array}{l}\text { Unruhe, Anspannung, Stress, Burnout, ADHS, } \\
\text { Nervenschwäche }\end{array}$ & $\begin{array}{l}\text { Nr. } 2 \text { Calcium phopshoricum } \\
\text { Nr. } 5 \text { Kalium phosphoricum } \\
\text { Nr. } 7 \text { Magnesium phosphoricum }\end{array}$ & Nr. 7 \\
\hline Entzündung & $\begin{array}{l}\text { chronische Entzündungen, Rheuma, } \\
\text { Sehnenscheidenentzündung, Schleimhaut- } \\
\text { entzündung }\end{array}$ & $\begin{array}{l}\text { Nr. } 3 \text { Ferrum phosphoricum } \\
\text { Nr. } 6 \text { Kalium sulfuricum } \\
\text { Nr. } 9 \text { Natrium phosphoricum }\end{array}$ & Nr. 3 \\
\hline Festtag & $\begin{array}{l}\text { bei üppigem Essen: fördert die Fettverdauung, } \\
\text { beeinflusst den Säure-Basen-Haushalt und die } \\
\text { Ausscheidung. Hilft auch bei Blähungen }\end{array}$ & $\begin{array}{l}\text { Nr. } 6 \text { Kaliumsulfuricum } \\
\text { Nr. } 9 \text { Natrium phosphoricum } \\
\text { Nr. } 10 \text { Natrium sulfuricum }\end{array}$ & Nr. 6 \\
\hline Kampf & $\begin{array}{l}\text { Müdigkeit, Konzentrationsschwierigkeiten } \\
\text { während Lernphasen, Kraftlosigkeit, } \\
\text { Erschöpfung, Motivationslosigkeit }\end{array}$ & $\begin{array}{l}\text { Nr. } 3 \text { Ferrum phosphoricum } \\
\text { Nr. } 5 \text { Kalium phosphoricum } \\
\text { Nr. } 8 \text { Natrium chloratum }\end{array}$ & Nr. 5 \\
\hline Rheuma & $\begin{array}{l}\text { Rheuma, Gicht, Arthrose, Gelenkbeschwerden, } \\
\text { Gelenkknacken }\end{array}$ & $\begin{array}{l}\text { Nr. } 8 \text { Natrium chloratum } \\
\text { Nr. } 9 \text { Natrium phosphoricum } \\
\text { Nr. } 10 \text { Natrium sulfuricum }\end{array}$ & Nr. 9 \\
\hline Schleimhaut & $\begin{array}{l}\text { allgemeine Beschwerden aller Schleimhäute, } \\
\text { z.B. Mund-, Magen- und Darmschleimhaut }\end{array}$ & $\begin{array}{l}\text { Nr. } 4 \text { Kalium chloratum } \\
\text { Nr. } 8 \text { Natrium chloratum } \\
\text { Nr. } 12 \text { Calcium sulfuricum }\end{array}$ & N. 12 \\
\hline Sport & $\begin{array}{l}\text { erhöht die Leistungsfähigkeit, vermindert } \\
\text { Krampfneigung, fördert die Regeneration }\end{array}$ & $\begin{array}{l}\text { Nr. } 3 \text { Ferrum phosphoricum } \\
\text { Nr. } 5 \text { Kalium phosphoricum } \\
\text { Nr. } 7 \text { Magnesium phosphoricum }\end{array}$ & Nr.5 \\
\hline Stoffwechsel & $\begin{array}{l}\text { Stoffwechselstörungen, Störungen des Leber- } \\
\text { Galle-Systems, Ausscheidungsstörungen, } \\
\text { unterstützend für Kuren }\end{array}$ & $\begin{array}{l}\text { Nr. } 8 \text { Natrium chloratum } \\
\text { Nr. } 9 \text { Natrium phosphoricum } \\
\text { Nr. } 10 \text { Natrium sulfuricum }\end{array}$ & Nr. 10 \\
\hline
\end{tabular}

oder Bettwäsche ist aufgrund der eventuellen Rückstände durch z.B. enthaltene Harze erst nach Prüfung empfehlenswert.

\section{Schüssler-Trio und Aromasprays}

Die langjährige Erfahrung mit der Anwendung von Schüssler-Salzen hat die Verwendung von sogenannten
Trios mit einem entsprechenden Aromaspray empfehlenswert gemacht [1]. Dabei werden je 3 Schüssler-Salze bei miteinander verwandten Indikationen bzw. Anwendungen mit einem entsprechenden Piniol Aromaspray kombiniert (Tab. 3).

Weiter hat sich ein "Grippe-Quattro» bei Erkältungs- und Grippesymptomen bewährt:
- Ferrum phosphoricum Nr. 3 plus

- Nr. 3 Ferrum phosphoricum

- Nr. 4 Kalium chloratum

- Nr. 10 Natrium sulfuricum

- Nr. 21 Zincum chloratum (Nr. 21 Zincum chloratum gehört zu den erweiterten Schüssler-Salzen)

Dazu wird der Aromaspray Nr. 3 verwendet. 